\title{
La Chartreuse de Parme. Stendhal, M. Crouzet (dir.)
}

\author{
Michel Arrous
}

\section{(2) OpenEdition}

\section{Journals}

\section{Édition électronique}

URL : http://journals.openedition.org/studifrancesi/9990

DOI : ERREUR PDO dans /localdata/www-bin/Core/Core/Db/Db.class.php L.34 : SQLSTATE[HY000]

[2006] MySQL server has gone away

ISSN : 2421-5856

\section{Éditeur}

Rosenberg \& Sellier

\section{Édition imprimée}

Date de publication : 1 août 2017

Pagination : $371-372$

ISSN : 0039-2944

\section{Référence électronique}

Michel Arrous, "La Chartreuse de Parme. Stendhal, M. Crouzet (dir.) », Studi Francesi [En ligne], 182 (LXI I II) | 2017, mis en ligne le 01 août 2017, consulté le 07 janvier 2021. URL : http://

journals.openedition.org/studifrancesi/9990; DOI : https://doi.org/10.4000/studifrancesi.9990

Ce document a été généré automatiquement le 7 janvier 2021.

\section{(c) (i) () $९$}

Studi Francesi è distribuita con Licenza Creative Commons Attribuzione - Non commerciale - Non opere derivate 4.0 Internazionale. 


\title{
La Chartreuse de Parme. Stendhal, M. Crouzet (dir.)
}

\author{
Michel Arrous
}

\section{RÉFÉRENCE}

La Chartreuse de Parme. Stendhal, actes du colloque international de Paris IV-Sorbonne, 23-24 novembre 1996, nouvelle édition, études réunies par Michel CROUZET, Paris, Eurédit, 2016, $371 \mathrm{pp}$.

1 Reprint opportun, car en 1997, date de la première édition, peu de recensions avaient signalé ces contributions fort variées puisqu'elles concernent la réception de l'œuvre, l'étude des sources et l'analyse thématique, aussi bien que la poétique. Avec L'"Origine" (pp. 7-25), Mariella DI MAIO dépasse la recherche érudite menée par Benedetto pour étudier les rapports du roman avec les récits italiens (l'Origine des grandeurs de la famille Farnèse), de la première ébauche rédigée par Stendhal (La jeunesse d'Alexandre Farnèse) à la réécriture ou plutôt à la réinvention qui le conduira à «change[r] Alexandre en Fabrice». Richard BOLSTER (pp. 27-41) relit le dernier chapitre et s'arrête sur la place de l'amour et le rôle déterminant de la mort de Sandrino. Cet élément pathétique, absent de la chronique au dénouement heureux et banal, ressemble à l'Histoire d'Éléonore de Parme, épisode d'un roman anglais que Stendhal avait apprécié en 1810. Pour Annalisa BOTTACIN, le thème de la passion est intimement lié à la puissance émotionnelle du lac, le paysage lacustre étant par excellence un des luoghi ameni (Le lac de Côme, paysage de l'âme, pp. 43-60). Dans ce roman de la passion, de la tendresse et du bonheur, il y a des zones d'ombre ou de silence: qu'en est-il de la sincérité et de la bonne foi? Dans $L a$ Sanseverina: amour et silences (pp. 61-70), Maurice MÉNARD montre que chez Gina elles sont quelques peu voilées ou menacées quand l'amour est en jeu. À la différence de son père, Stendhal n'eut aucune passion pour Bourdaloue et Massillon, et pas davantage pour Bossuet et autres prêcheurs, à l'exception de Fénelon. Néanmoins ses connaissances se retrouvent dans le talent et la carrière du prédicateur amoureux de 
Clélia qu'analyse Rosa GHIGO BEzzoLA, un prédicateur voué à la religion de l'amour (Les sermons de «monsignore» Fabrice Del Dongo, pp. 71-85). Tout aussi mondaine, mais cette fois selon des codes ludiques, la stratégie que Pierrette-Marie NEAUD déchiffre dans la pratique du jeu politique dirigé par Mosca et la Sanseverina (Les règles d'une partie de whist ou le jeu politique à la cour de Parme, pp. 87-102). Christof WEIAND propose une analyse phénoménologique de la temporalité en distinguant le «temps du raconter» du «temps raconté», le temps dynamique du temps statique (L'esthétique du temps dans "La Chartreuse de Parme", pp. 103-118). Retour à la réception de l'œuvre par le biais de l'exemplaire sur lequel Stendhal consigna la plupart des corrections suscitées par la lecture de la «Revue parisienne». Patrick BERTHIER (Stendhal jugé par Balzac. Les enseignements de l'exemplaire "Chaper» de "La Chartreuse de Parme", pp. 119-127) fait état des perplexités de Stendhal et du mal qu'il eut à répondre à Balzac, un Stendhal qui n'aurait sans doute pas recommencé La Chartreuse. Suit, mais on ne s'en étonnera pas, un véritable essai de Michel CROUZET sur "La Chartreuse de Parme", roman de l'euphémisme (pp. 129-171): dans la patrie de la force et du beau, le grand roman italien progresse selon une «stratégie de mise à distance, d'endiguement, de résistance, non de suppression du négatif». Le roman est marqué par un euphémisme généralisé, comme on le voit dans le traitement du motif de la prison, mais, pour autant, les laideurs et les bassesses ne sont pas escamotées. Ce roman est aussi un roman sous le signe de l'imprévu, dans l'épisode de Waterloo comme dans les aventures amoureuses de Fabrice. Ann JEFFERSON suit les grandes péripéties de l'intrigue entraînant soit des quiproquos, soit des duperies ou des maladresses aux conséquences plus ou moins néfastes (Erreur et quiproquo dans "La Chartreuse de Parme", pp. 173-184). On sait que dans la perception et l'esthétique stendhaliennes, les rôles du détail et de la physionomie générale s'équilibrent. Marghareta LEONI rappelle que ce double mouvement se lit déjà dans l'Histoire de la peinture et montre que dans le roman la vue du détail coloré se combine à celle des lointains voilés (La physionomie et le détail, le voile et la couleur dans "La Chartreuse de Parme", pp. 185-204). Dans ce récit si complexe, Mariane BURY privilégie la dimension de la simplicité (Le choix du «simple», pp. 205-216). Chez Stendhal la simplicité est un idéal à la fois esthétique et moral («faire simple c'est être soi») illustré par la simplicité de Fabrice et la simplicité du style. La musique et les larmes dans "La Chartreuse de Parme" (pp. 217-230): Suzel ESQUIER aborde d'une nouvelle manière la question de la musicalisation d'un roman qui unit le désir et la mélancolie à la musique. De la musicalité au lyrisme, il n'y a qu'un pas que se garderait de franchir l'idéologue; Jean SARROCHI le dit tout en pratiquant non sans malice l'art du contre-pied (Illyrique "Chartreuse", pp. 231-247). Stendhal affligé d'impuissance lyrique? Plutôt partisan de l'ellipse, comme le confirment quelques affleurements, quelques «nappes» lyriques. Dans son Analyse structurale du roman (pp. 249-263), Hans BOLL-JOHANSEN met en évidence les relations d'opposition (passion et vanité, Sud et Nord, action intérieure et action extérieure, répartition des personnages féminins, héros et adversaires). Pierrette PAVET, qui les a plus tard analysés en détail dans sa thèse (Stendhal et le ridicule, Eurédit, 2013), évoque les nuances et les contrastes du ridicule ostentatoire, à la cour de Parme, et du ridicule intime, qui concerne principalement Fabrice (pp. 265-277). Si l'échec de ses tentatives théâtrales explique en partie le passage de Stendhal au roman, on peut aussi dire que la comédie s'est déplacée dans le roman ou, mieux, que le roman a remplacé la comédie. Dans le roman parmesan, comme déjà dans le Rouge, abondent les scènes, si bien que Daniel SANGSUE affirme que le ridicule règne, non sans se mêler au 
tragique ("La Chartreuse de Parme" ou la comédie du roman, pp. 279-293). Ce «livre donné » (Gracq) est le fruit d'une patiente réflexion sur le romanesque qui, tel que le conçoit Stendhal, repose sur des situations d'opposition entre liens et passions. Cette dramatisation des passions, Stendhal l'a rencontrée dans le modèle classique du roman d'amour, mais aussi chez «son grand ennemi» Chateaubriand. Fabienne BERCEGOL mentionne la dette de Stendhal à l'égard du Génie du christianisme, soigneusement lu, réinterprété voire altéré. De cette poétique des passions, on a une belle illustration avec les combats intérieurs de la chrétienne Clélia, déchirée entre son amour pour Fabrice et son devoir filial, entre sa foi (le souci de fidélité au vœu fait à la Madone) et sa passion (Clélia ou le romanesque des "combats intérieurs de l'âme», pp. 295-310). Béatrice DIDIER signale un aspect du romanesque stendhalien auquel les lecteurs ne prêtent pas toute l'attention requise, à savoir l'échange épistolaire qui se développe au fil du roman. D'où l'intérêt d'examiner ce phénomène en tenant compte de l'emplacement des lettres et billets, de leur situation dans l'intrigue, de leur répartition selon les personnages («Écris [...] toi qui as une main droite», pp. 311-324). À contre-courant, mais c'est tant mieux, d'une tradition vivace dans les études stendhaliennes, Didier PHILIPPOT reprend la question du «point de vue» ou de la focalisation, à partir de l'épisode de Waterloo considéré, depuis Blin, comme le paradigme de la narration stendhalienne (Le «réalisme subjectif» dans "La Chartreuse de Parme": une idée reçue?, pp. 325-352). Dans ce célèbre épisode, l'emploi du réalisme subjectif est un fait exceptionnel car dans les autres grands moments de l'action (notamment lors de l'évasion) Stendhal abandonne l'optique subjective au profit de la saisie par l'extérieur: «Fabrice est alors le centre de l'action sans être le centre de vision». Dans la narration stendhalienne il n'y a pas de forme fixe; le point de vue varie sans cesse et la restriction de champ n'empêche pas l'omniscience. Si dans l'épisode de Waterloo le récit privilégie la vision et la conscience de Fabrice, l'auteur ne s'absente pas qui est "présent par l'ironie». Enfin, il y a le jeu entre folie, rire et politique, trinité qui retient Nathalie PREISs ("La Chartreuse de Parme": chartreuse ou folie? Folie, rire et politique, pp. 353-365). Folie en tout genre à la cour de Parme, du risible à l'odieux, sans oublier le grotesque de la politique quand la peur devient raison d'État. 